\title{
Expectativas y percepciones sobre calidad del servicio educativo
}

\author{
Julio César Sánchez Sánchez ${ }^{1}$
}

\section{Resumen}

Esta investigación busca analizar las expectativas y las percepciones de los aprehendientes sobre la calidad del servicio que ofrece la UNAD en Cartagena. Se parte de que calidad hace referencia a propiedades y características de un bien o servicio frente a las necesidades del consumidor, y es relativa, por cuanto cada individuo tiene una percepción diferente de ella.

Si el bien o servicio colma las expectativas de los consumidores se aseguran la fidelidad hacia la organización y la permanencia de esta en el mercado; por tanto, es importante conocer qué esperan los consumidores y que percepción tienen una vez adquirido el bien o recibido el servicio.

Para medir la calidad se utiliza la metodología SERVQUAL, con instrumentos de entrada y salida, y que contempla los ítems, los elementos tangibles, la fiabilidad, la capacidad de respuesta, la seguridad y la empatía a partir de los cuales se plantean preguntas con respuestas cuantitativas tabuladas de 1 a 5 .

Aplicados los instrumentos, estos dan una valoración general de 4,6 sobre 5,0 para las expectativas, y de 3,8 sobre 5,0 para las percepciones. La diferencia o brecha (gap) se debe analizar, pues tendría que tender a cero (0), lo cual equivale a afirmar que el consumidor percibe un servicio en los términos en los cuales esperaba recibirlo.

Palabras clave: Calidad, educación, expectativa, percepción, satisfacción, servicio.

\section{Abstract}

This research seeks to analyze the expectations and perceptions of learners on the quality of service offered by UNAD in Cartagena. It is assumed that quality refers to the properties and characteristics of possessions or services meet the needs of the consumer, is relative because each person has a different perception of it.

If the possessions or service fulfills the expectations of consumers ensures loyalty to the organization and the remain on the market, therefore, it is important to know what consumers expect and what perception has once acquired the possessions or service received.

To measure quality using the SERVQUAL methodology with tools that includes input and output items, tangible elements, Reliability, response capacity, Safety and empathy from which it posed questions with quantitative answers tabulated from 1 to 5 .

Applied instruments they give an overall rating of 4.6 on 5.0 for expectations and 3.8 on 5.0 for perceptions. The difference or gap (GAP) should be analyzed because they must tend to zero (0) which is equivalent to affirm that the consumer receives a service on the terms in which it expected to receive.

Key words: Quality, education, expectation, perception, satisfaction, service.

Recibido: 3 de mayo de 2012

Aceptado: 27 de julio de 2012

${ }^{1}$ Economista. Msc. en administración. Universidad Nacional Abierta y a Distancia - (UNAD). Correo electrónico: julio.sanchez@unad.edu.co 


\section{Introducción}

El concepto de calidad en la educación superior es fundamental para el desarrollo de los países, pues son las universidades las que preparan a los profesionales que en el futuro dirigirán los destinos de los mismos países, ya sea como funcionarios públicos o como empresarios.

En cuanto se considera que "La educación superior ha sido cada vez más reconocida como una industria de servicios la cual debe esforzarse para identificar las expectativas y necesidades de sus clientes, que son los Estudiantes" (Mello, Dutra y Oliveira, 2001, p. 130), se requiere, por tanto, conocer qué esperan recibir dichos clientes y qué nivel de importancia le otorgan a cada uno de los elementos que tienen relación directa o indirecta con la prestación del servicio que se recibe.

Para el caso de la UNAD, dada su estructura, conformada por 5 escuelas, 8 zonas y 56 centros, la investigación se podrá aplicar de manera individual en cada ubicación, y, escalonadamente, hasta hacerlo en toda la organización, si así se requiere.

Los resultados que aquí se presentan son producto de la aplicación de los instrumentos de entrada y salida (expectativas y percepciones) a los estudiantes que ingresaron por primera vez durante el segundo periodo de 2011 a todos los programas de la Escuela de Ciencias Administrativas, Contables, Económicas y de Negocios - (ECACEN) - en el CEAD Simón Bolívar, de Cartagena de Indias; es decir, un total de 53 estudiantes.

\section{Objetivos}

\section{General}

Analizar las expectativas y las percepciones que tienen los aprehendientes sobre los servicios ofrecidos por la Universidad Nacional Abierta y a Distancia (UNAD) en el CEAD Simón Bolívar de Cartagena de Indias.

\section{Específicos}

- Identificar los elementos que componen cada uno de los ítems de estudio según la metodología SERVQUAL.

- Identificar las expectativas con que ingresan los estudiantes a la Universidad Nacional Abierta y a Distancia UNAD en el CEAD Simón Bolívar de Cartagena de Indias.

- Identificar las percepciones de los estudiantes de la Universidad Nacional Abierta y a Distancia UNAD en el CEAD Simón Bolívar de Cartagena de Indias una vez han recibido el servicio.

- Contrastar las diferencias entre las expectativas y las percepciones identificadas.

\section{Fundamentación teórica}

Según Lovelock (2001), el servicio de la educación está clasificado como un servicio con acciones intangibles, dirigida a las mentes de las personas y que se realiza con una entrega continua y se lleva a cabo a través de una asociación entre la empresa que presta el servicio y su cliente.

Las empresas deben dirigir sus acciones a producir bienes o prestar servicios de tal modo que las expectativas de los clientes sean cumplidas y superadas, para así buscar ser las preferidas y mantenerse en el mercado, pues "la calidad es generalmente considera un atributo en la elección de los consumidores" (Bateson, 2001, p. 363).

La calidad se juzga según la satisfacción percibida. Según Grönroos (2005, p.54), la calidad percibida se determina "por la diferencia entre la calidad esperada y de calidad con experiencia"; o sea, es la diferencia entre las percepciones de los clientes al recibir el servicio y las expectativas con las cuales ellos abordaron la prestación del mismo servicio.

Satisfacer las expectativas inmediatas y explicitas de los clientes debe buscarse en el corto plazo. Sin embargo, a mediano y largo plazo es importante desarrollar las competencias para alcanzar las metas de suplir las necesidades reales, sean estas explicitas o inconscientes (Coelho, 2004, p 37). Según el mismo Coelho, la calidad solo se mide al final del proceso; es decir, cuando el servicio se ha prestado y no hay manera de cambiar la percepción del cliente sobre lo que ha recibido.

Tanto la investigación académica como la práctica empresarial vienen sugiriendo, desde hace algún tiempo, que un elevado nivel de calidad de servicio proporciona a las empresas considerables beneficios en cuanto a cuota de mercado, productividad, costes, motivación del personal, diferenciación respecto a la competencia, lealtad y captación de nuevos clientes. Como resultado de dicha evidencia, la gestión de la calidad de servicio se ha convertido en una estrategia prioritaria, y cada vez son más quienes tratan de definirla, medirla y, finalmente, mejorarla. Sin embargo, la definición y la medida de la calidad han resultado ser particularmente complejas en el ámbito de los servicios, pues al hecho de que la calidad sea un concepto aún sin definir claramente cabe añadirle la dificultad derivada de la naturaleza intangible de los servicios (Grönroos,1994).

Aún así, la calidad se ha convertido en pieza clave dentro del sector terciario, y su búsqueda ha llevado a numerosos investigadores a desarrollar posibles definiciones y a diseñar modelos sobre ella (Buttle, 1996). En la literatura sobre el tema el modelo que goza de mayor difusión es el denominado Modelo de las Deficiencias 
(Parusaraman, Zeithaml y Berry, 1985, 1988), en el que se define la calidad de servicio como una función de la discrepancia (gap) entre las expectativas de los consumidores sobre el servicio que van a recibir y sus percepciones sobre el servicio efectivamente prestado por la empresa.

\section{Métodos}

El presente trabajo es de tipo descriptivo, por cuanto busca indagar sobre las expectativas de quienes ingresan por primera vez a los diferentes programas de la escuela ECACEN, de la Universidad Nacional Abierta y a Distancia (UNAD) en el CEAD Simón Bolívar de Cartagena de Indias; es explicativo en tanto indaga sobre cómo perciben los estudiantes los servicios educativos recibidos y los contrasta con las expectativas con las cuales llegaron, para encontrar las posibilidades de mejora.

Para su realización, se toma como universo la totalidad de los estudiantes que ingresan por primera vez a los programas de la escuela ECACEN de la UNAD en el CEAD Simón Bolívar de Cartagena de Indias durante el segundo periodo de 2011, y que fueron, en total, 53 estudiantes.

En primera instancia se debe analizar lo concerniente a las expectativas con las cuales llegan los consumidores a que se les preste el servicio, y que para este caso son los estudiantes recién llegados a la Universidad. Este análisis, según la metodología SERVQUAL, se realiza trabajando cinco categorías: a) Elementos tangibles; b) Fiabilidad; c) Capacidad de respuesta; d) Seguridad, y e) Comprensión empática.

Dentro de cada una de tales categorías se elaboran los cuestionarios con las preguntas sobre las cuales se requiere indagar, y que muestran cuál es el nivel de importancia que tienen para el consumidor. El resultado muestra qué elementos son relevantes para el consumidor y en que medida. Cada pregunta tiene una escala de valoración numérica y su equivalente cualitativo, así:

\section{$1=$ Nada importante}

2=Un poco importante

3=Medianamente importante

\section{$4=$ Importante}

\section{$5=$ Muy importante}

El tipo de pregunta se hace libremente por cada organización, de acuerdo con lo que se desea indagar en cada categoría, y el número de preguntas oscila entre cinco y diez preguntas por categoría. Para el caso de la presente investigación se trabajaron las siguientes preguntas:
- Elementos tangibles: tiene que ver con la apariencia de los elementos físicos, los equipos, el personal y los materiales de comunicación de la organización, así: 1) Instalaciones físicas; 2) Decoración interna; 3) Espacios deportivos y recreativos; 4) Servicio de cafetería ; 5) Cantidad de equipos de informática; 6) Velocidad de navegación; 7) Apoyo de los funcionarios; 8) Centro de copiado y papelería; 9) Equipo de docentes; 10) Oficina de orientación.

- Fiabilidad: se relaciona con todas las situaciones que le generan confianza al estudiante: 11) Inmediatez de la respuesta; 12) Satisfacción con la respuesta; 13) Compromiso y tiempo en las comunicaciones; 14) Apoyo presencial; 15) Respeto por las fechas de inicio y terminación del periodo académico.

- Capacidad de respuesta: hace referencia a situaciones que tienen que ver con la forma como responde la institución a los requerimientos del estudiante: 16) Capacidad local para resolver problemas; 17) Capacidad de los funcionarios para resolver problemas; 18) Dar tiempos específicos para resolver problemas; 19) Capacidad de los funcionarios para direccionar hacia quien puede resolver un problema determinado; 20) Claridad en la información entregada.

- Seguridad: relacionado con todo lo que genera sensación de seguridad en el usuario: 21) Labor de los funcionarios de vigilancia; 22) Existencia de cámaras de seguridad; 23) Existencia de señalización y rutas de evacuación en la edificación; 24) Existencia de enfermería; 25) Estado de la iluminación en aulas y pasillos.

- Comprensión empática: hace referencia a la relación que se genera entre los funcionarios y los estudiantes como consecuencia del trato durante la prestación del servicio: 26) Disposición de los funcionarios a escuchar y responder; 27) Vocabulario y tono de voz de los funcionarios; 28) Sistema de atención al estudiante; 29) Conocimiento del área de desempeño por parte de los funcionarios; 30 ) Trato de los funcionarios hacia los clientes.

En segunda instancia, y teniendo como base las cinco categorías anteriormente descritas, una vez el estudiante ha recibido el servicio y utilizando la esencia de las mismas preguntas para medir las expectativas, se procede a aplicar otro cuestionario, con el fin de indagar sobre cómo percibió cada uno de esos elementos; también se le da a ello una valoración numérica y su correspondiente cualitativo, así:

1=Muy malo (a)
2=Malo (a)
3=Regular
4=Bueno (a)
5=Muy bueno (a)


La diferencia de resultados obtenidos en cada una de las preguntas y cada uno de los ítems entre la primera y la segunda instancias marcan claramente dónde se debe mejorar, y en qué medida, para llegar a responder adecuadamente y suplir las necesidades de los consumidores tal como ellos lo esperan.

Obtenida la información correspondiente a la aplicación de las encuestas, se procede a su tabulación aplicando el programa Statgraphics, con el fin obtener los resultados y realizar el análisis correspondiente.

\section{Análisis de resultados}

Aplicados los instrumentos para recolectar información, y con base en estadísticos básicos, se encontró lo siguiente:

\section{Visión general}

En cuanto a las expectativas, se obtiene, en términos generales, una valoración numérica de 4,6 sobre 5,0, lo que equivale a una valoración cualitativa cercana a Muy importante. Una vez recibidos los servicios, la valoración numérica de los estudiantes es de 3,8 sobre 5,0, equivalente a una valoración cualitativa de Importante. La brecha, o gap, es de $-0,8$, y representa lo que se debe mejorar (Gráfico 1).

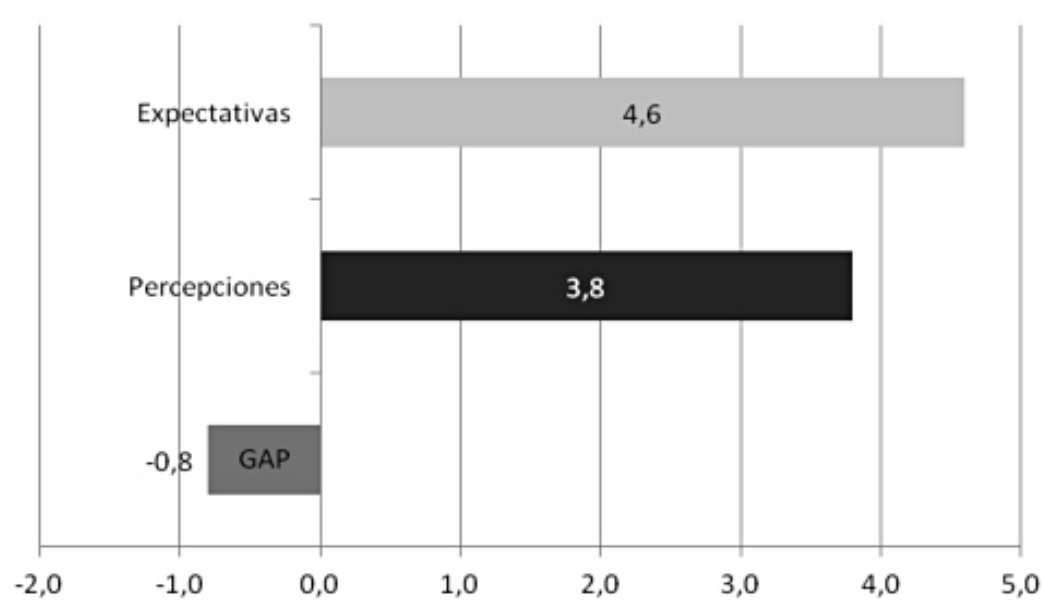

Gráfico 1. Valoración general de expectativas y las percepciones

Fuente: elaborado por el autor.

\section{Valoración de ítems}

En una primera desagregación se encuentran las expectativas y las percepciones de cada uno de los cinco ítems que propone la metodología de estudio (Gráfico 2). El mayor gap se encuentra en el ítem seguridad $(-1,6)$, seguido del ítem elementos tangibles $(-1,1)$; los demás ítems tienen unos gap relativamente bajos $(-0,2$; $-0,3 ;-0,4)($ Gráfico 2). La valoración de percepción más baja se encuentra en el ítem seguridad $(2,9)$, frente a una valoración de expectativas de 4,5. Los elementos que más inciden para obtener esta valoración tan baja se analizarán en una segunda desagregación.

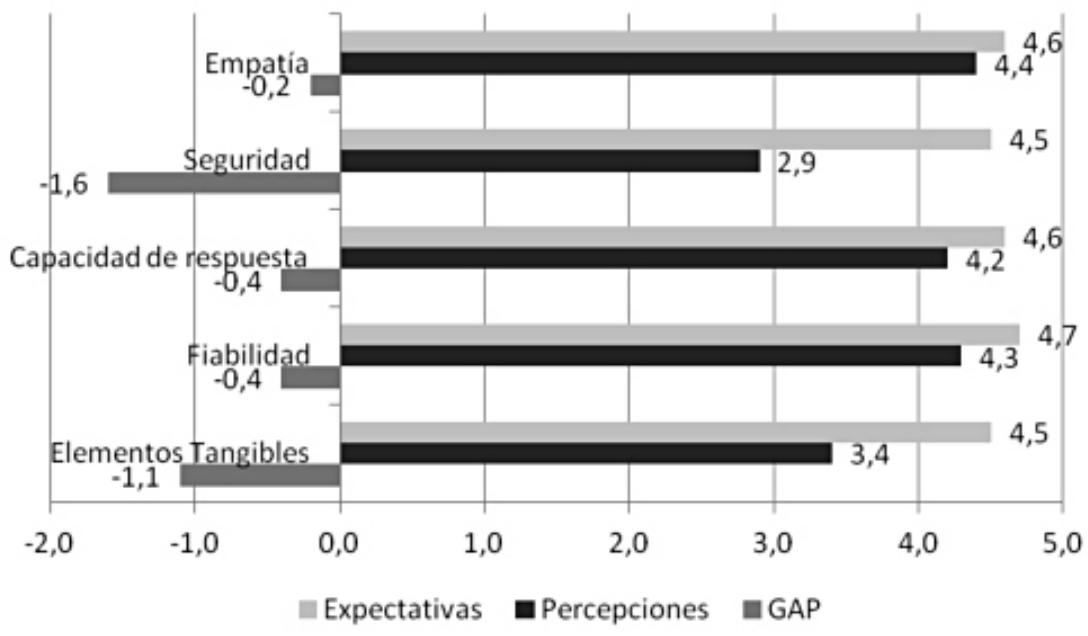

Gráfico 2. Valoración por ítems

Fuente: elaborado por el autor. 
Una segunda desagregación permite observar los resultados individuales por ítem y el aporte de cada uno de los elementos a cada ítem.

\section{Valoración del ítem Elementos tangibles}

En cuanto al ítem elementos tangibles (Gráfico 3), que tiene el segundo mayor gap $(-1,1)$, los elementos que tienen mayor incidencia en tal diferencia son el estado de las instalaciones físicas, con un gap de -1,6, lo cual se puede justificar por el hecho de estar la edificación en proceso de remodelación y estarse prestando el servicio en esas condiciones.
Un segundo elemento lo componen los espacios deportivos y recreativos, con un gap de $-1,6$. El tercer elemento es la carencia de un centro de copiado y papelería, con un gap de -1,5; los elementos decoración interna de la universidad y número de equipos de informática aportan un gap relativamente alto $(-1,3)$, seguido muy de cerca por los servicios de cafetería, con un gap de $-1,2$. El elemento de menor gap $(-0,2)$ es el equipo de consejeros, tutores y docentes de apoyo.

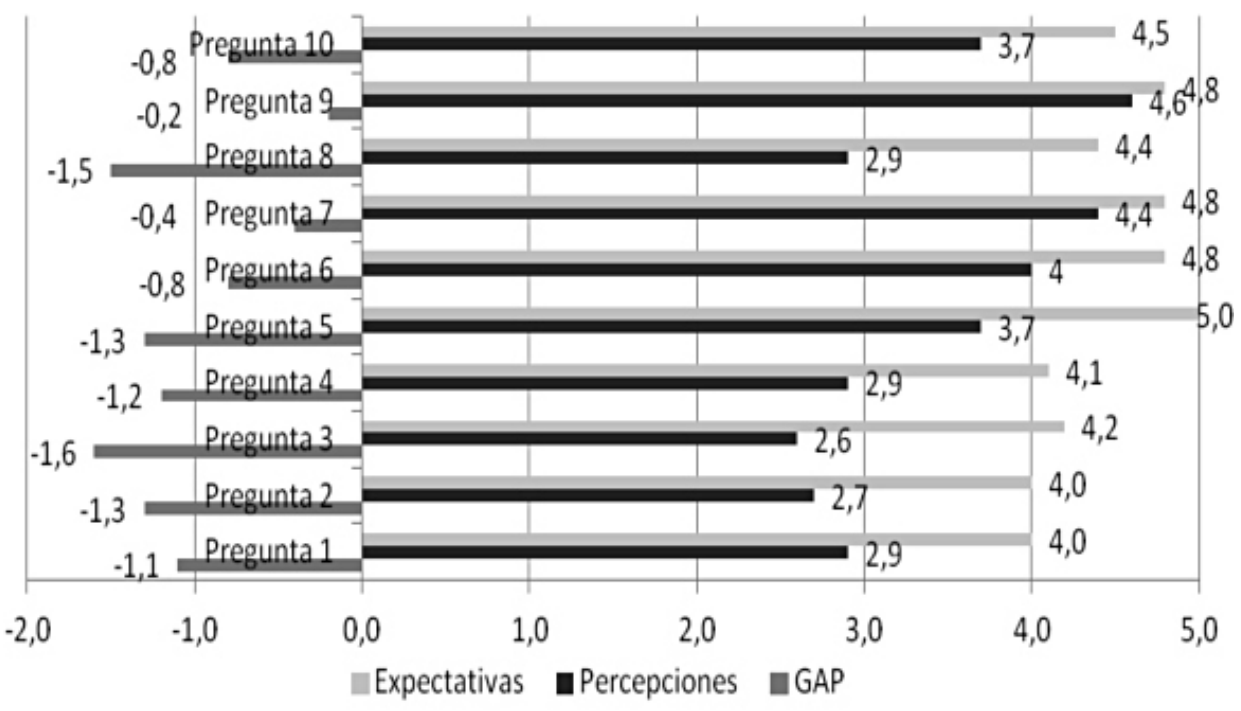

Gráfico 3. Valoración del ítem Elementos tangibles

Fuente: elaborado por el autor.

\section{Valoración del ítem Fiabilidad}

El ítem fiabilidad presenta un bajo gap $(-0,4)$, debido a que sus elementos constitutivos también presentan resultados bastante bajos (Gráfico 4). El más significativo es el elemento inmediatez de respuesta por parte de los estudiantes, el cual tiene un gap de -0,7. Los estudiantes llegan con unas expectativas valoradas en 4,8; es decir, para ellos es de mucha importancia este ele- mento, y en percepción lo valoran en 4,1 , que equivale a Bueno. El siguiente elemento es el tiempo estipulado y oportuno para que los funcionarios den respuesta a los requerimientos de los estudiantes, y el cual presenta un gap de -0,5; los estudiantes valoran este elemento en 4,8 , lo que equivale a Muy importante, y la valoración de lo que perciben es de 4,3; apenas ligeramente por encima de Bueno. 


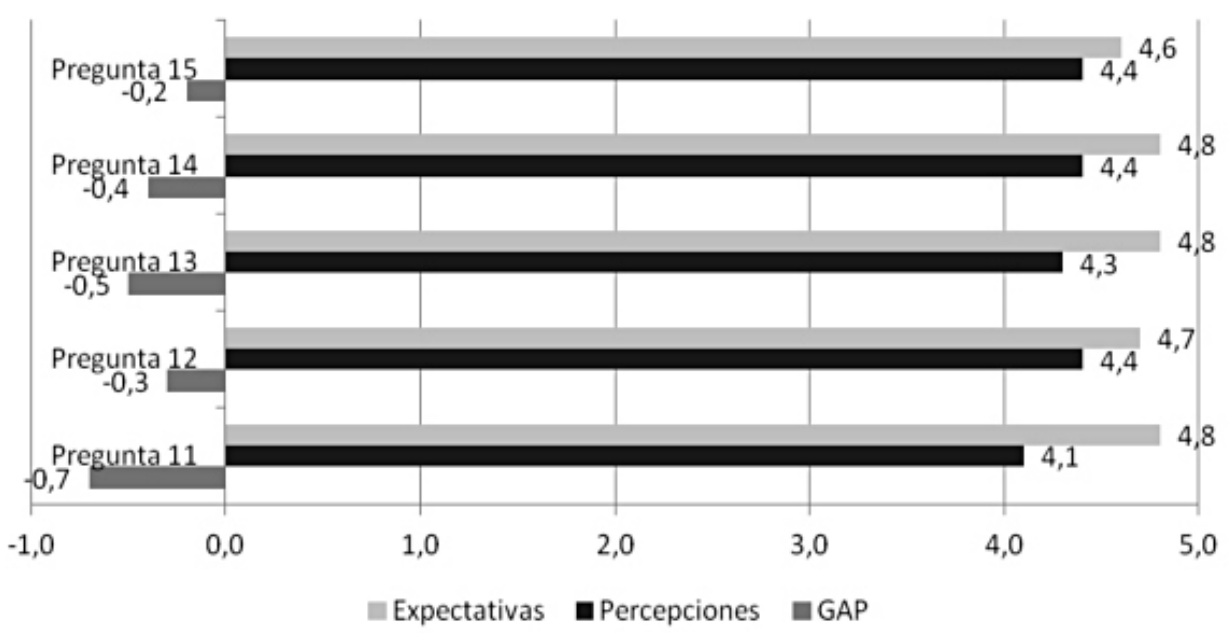

Gráfico 4. Valoración del ítem Fiabilidad

Fuente: elaborado por el autor.

\section{Valoración del ítem capacidad de respuesta}

El ítem Capacidad de respuesta presenta un gap de -0,3; el elemento Solución directa por parte del CEAD de los inconvenientes presentados es el que tiene el

mayor gap: -0,6; le sigue el elemento Claridad y facilidad para entender las respuestas dadas, con un gap de $-0,5$. El elemento tiempo de respuesta presenta un gap de 0,0 (Gráfico 5).

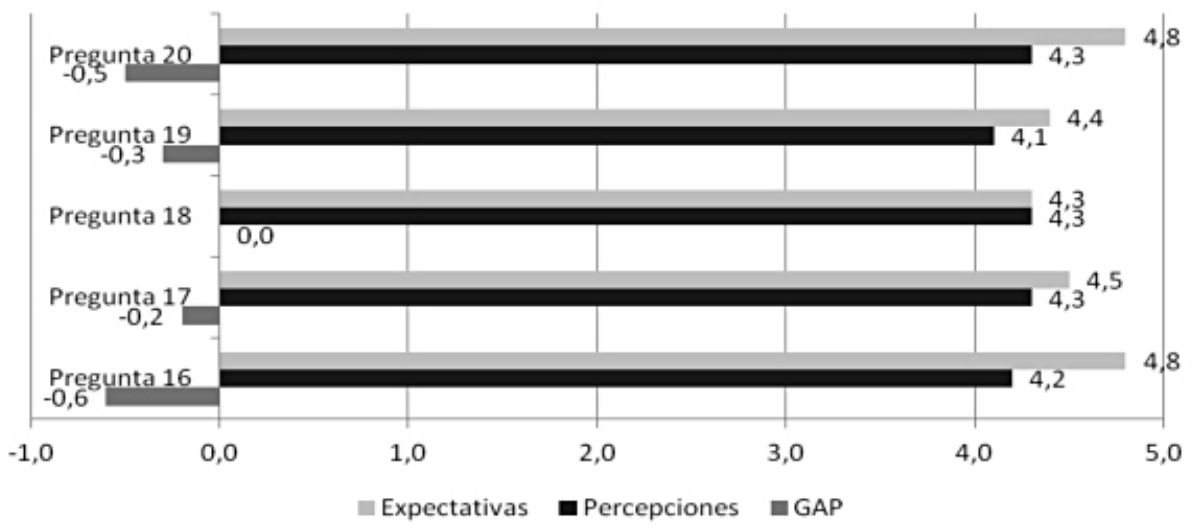

Gráfico 5. Valoración del ítem Capacidad de respuesta.

Fuente: elaborado por el autor.

\section{Valoración del ítem Seguridad}

El ítem Seguridad es el que presenta un mayor gap: $-1,6$, como producto del aporte de cada uno de los elementos que lo componen, y dentro de los cuales el de mayor valor es el elemento Enfermería, con un gap de
$-2,5$. Para los alumnos es muy importante disponer de una enfermería, y la carencia de ella genera una percepción de inseguridad muy alta; le siguen los elementos Cámaras de seguridad e lluminación, con un gap de -1,7; luego está el elemento Señalización, con un gap de -1,6 (Gráfico 6). 


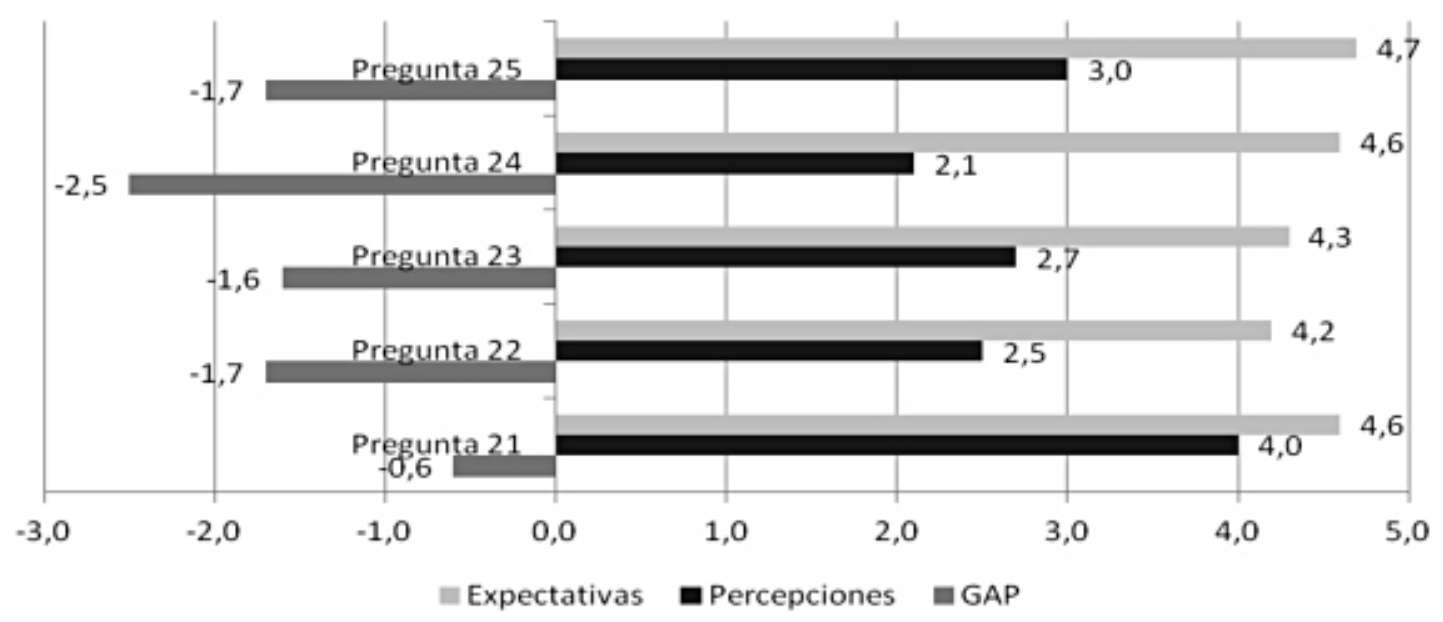

Gráfico 6. Valoración del ítem Seguridad

Fuente: elaborado por el autor.

\section{Valoración del ítem Empatía}

El ítem Empatía presenta un gap relativamente bajo $(-0,2)$; respecto a él, el elemento de mayor importancia fue la disposición, por parte de los funcionarios, para escuchar y responder a las solicitudes presentadas por los estudiantes. En este ítem también se incluye un elemento con gap de 0,0, correspondiente al elemento Atención individualizada que reciben los estudiantes cuando lo requieren (Gráfico 7).

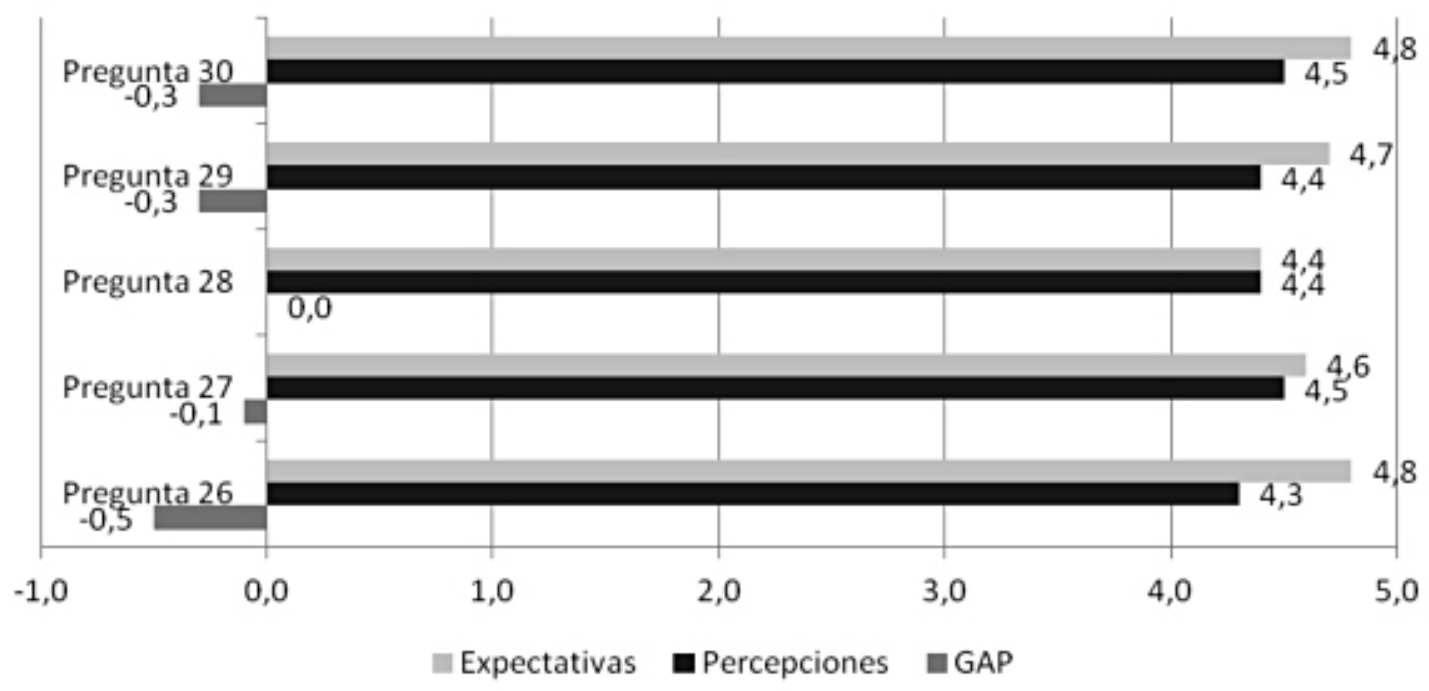

Gráfico 7. Valoración del ítem Empatía

Fuente: elaborado por el autor.

\section{Conclusiones}

Realizado el ejercicio de identificar las expectativas con las cuales ingresan los estudiantes de la escuela ECACEN, de la Universidad Nacional Abierta y a Distancia (UNAD) en el CEAD Simón Bolívar de Cartagena, y contrastadas dichas expectativas con las percepciones que tuvieron una vez recibido el servicio educativo, se puede concluir lo siguiente:
Los instrumentos aplicados permitieron identificar las expectativas con las cuales llegaron los estudiantes que ingresaron por primera vez a los programas de la escuela ECACEN en el CEAD Simón Bolívar de Cartagena de Indias, así como conocer las percepciones que tuvieron sobre los servicios que se les prestaron.

Los estudiantes que ingresaron por primera vez a los programas de la escuela ECACEN de la Universidad Nacional Abierta y a Distancia (UNAD) en el CEAD Si- 
món Bolívar de Cartagena esperan recibir unos servicios educativos cuyos componentes, en términos cualitativos, consideran "Muy importantes", con una valoración numérica de 4,6 sobre 5,0. Una vez recibidos los servicios, la percepción de los recibidos pasa a ser valorada cualitativamente como "Buena," con una calificación de 3,8 sobre 5,0; esto arroja una diferencia, brecha o gap de $-0,8$, que puede considerarse, a primera vista, no muy preocupante, pero la cual es necesario analizar de manera desagregada, para conocer en detalle dónde se ubican las oportunidades de mejora.

De los cinco ítems que componen el estudio (Elementos tangibles, Fiabilidad, Capacidad de respuesta, Seguridad, y Empatía), se encuentran dos en los cuales hay que tener especial cuidado, dada la alta valoración del gap; ellos son: en primer lugar, el ítem Seguridad, con un gap de -1,6, y en segundo lugar, el ítem Elementos tangibles, con un gap -1,1.

La alta valoración del gap en el ítem Seguridad se debe a que hay elementos con una gran necesidad de mejora, como ante el caso de la existencia de una enfermería que tiene la diferencia mas grande (gap -2,5; (Gráfico 6), lo cual es muy importante para las estudiantes, pero no se cuenta con ella; le siguen la existencia de cámaras de seguridad y la iluminación, lo cual también se valora como Importante y, muy importante por los estudiantes, pero a los que perciben como malo y Regular, respectivamente.

En cuanto al ítem Elementos tangibles, que tiene la segunda diferencia más grande (gap -1,1), los elementos más necesitados de atención son el estado de las instalaciones físicas y los espacios deportivos y recreativos, que tienen un gap de $-1,5$, pero que, seguramente, se solucionaran con la culminación del proceso de remodelación al cual esta siendo sometida la edificación de la universidad.

El tercer elemento por mejorar incluye la decoración interna de la universidad y la cantidad de equipos de informática con los que cuenta esta; elementos que presentan un gap de -1,3. La cafetería es otro elemento para mejorar, pues presenta un gap de $-1,2$.

El ítem que cuenta con una menor diferencia (gap de -0,2) es el de Empatía, lo que demuestra una muy buena relación entre los funcionarios y los estudiantes.

\section{Referencias bibliográficas}

Arapé, J. (1999). Estrategia, medición y calidad total. Recuperado de http://www.visiongc/Documentos/bsc los sistemas de medidas blandas y la calidad total.pdf.
Berry, L. Parasuraman, A. \& Zeithaml V. A. (1988). El servicio y la calidad. Madrid Editorial Horizonte.

Brunner, J. J. (1999). Los nuevos desafíos de la Universidad. Revista Electrónica de educación, Ciencia y Técnica, Universidad de Buenos Aires 91. Recuperado de educyt@de.fcen.uba.ar.

Dávila, J. M. (2001). Análisis de los factores determinantes de la calidad del servicio y su repercusión en la satisfacción del cliente y en la lealtad al proveedor: Estudio empírico en los establecimientos hoteleros de Castilla y León. Tesis doctoral. Universidad de León.

Grönroos, C. (2007). Service management and marketing: customer management in service competition. Chichester: John Wiley \& Son

López, M. C., Serrano, A. \& Sarabia, M. (2001). Una propuesta de modelo estratégico para la gestión de la calidad del servicio. Recuperado de empresa.unizar.es.

Lovelock, C. (2009). Marketing de Servicios. México: Pearson.

Oliveira, O. J. (2009). Adaptation and application of the SERVQUAL scale in higher education. São Paulo: UNESP-São Paulo State University.

Ruiz-Olalla, C. (2001). Gestión de la calidad del servicio. Recuperado de http://www.5campus.com/leccion/ calidadserv

Satolo, E., Ferreira, S., Cauchick, P., Araújo, F. (2005). Uma avaliação da qualidade em serviços em uma livraria e papelaria utilizando o SERVQUAL - um estudo exploratório. Ponencia presentada en el XXV Encontro Nacional de Engenharia de Produção-Porto Alegre.

Vásquez, R. \& Díaz, A. (2001). El conocimiento de las expectativas de los clientes: Una pieza clave de la calidad de servicio en el Turismo. Oviedo: Universidad de Oviedo.

Vásquez, R., Rodríguez, I. \& Díaz, A. (2001). Estructura multidimensional de la calidad de servicio en cadenas de supermercados: Desarrollo y validación de la escala CALSUPER. Recuperado de www.fade.es/faPag/webFade/infoempresa/.

Zanella, A., Lima, L. S. \& Lopes, L. F. D. (2006). Identificação de fatores que fluenciam na satisfação dos clientes de um clube recreativo por meio de análise fatorial. Revista GEPROS 1 (2) 